\title{
Distributed Sampling Rate Adaptation for Networked Control Systems
}

\author{
Jia Bai, Emeka P. Eyisi, Yuan Xue, Xenofon D. Koutsoukos \\ Department of Electrical Engineering and Computer Science, Vanderbilt University \\ Email: \{jia.bai, emeka.eyisi, yuan.xue, xenofon.koutsoukos\}@vanderbilt.edu
}

\begin{abstract}
Building networked control systems is a promising direction that promotes the evolution of the traditional control systems. The ability of using different sampling rates in control systems provides the flexibility for adapting their resource needs based on the dynamic networking environment. This paper studies the dynamic rate adaptation problem for networked control systems. In particular, we define a utility function which quantifies the relationship between the performance of a control system and its sampling rate. Then we formulate the rate adaptation problem as an optimal resource allocation problem, where the aggregated utility is maximized. We further present a price-based algorithm, where prices are generated to reflect the network utilization penalties and are used as the basis for rate adaptation. We formally prove the stability of our algorithm. The rate adaptation algorithm is further evaluated in an integrated simulation environment that consists of Matlab and ns-2, which allows highly accurate evaluation of network effects on the NCS performance. The experiment results show that our algorithm is able to provide agile and stable sampling rate adaptation.
\end{abstract}

\section{INTRODUCTION}

The integration of physical systems through computing and networking has become a trend now known as CyberPhysical Systems (CPS). Many CPS such as automotive vehicles and distributed robotics, are monitored and controlled by Networked Control Systems (NCS). These systems exchange information among sensors, controllers and actuators over a communication network. NCS are increasingly deployed over wireless networks, as they provide great convenience in terms of deployment and mobility support. In such a resource-constrained and dynamic networking environment, the capability of adaptive resource management is crucial for NCS to fully exploit the available resource and achieve desirable system performance.

This paper investigates sampling rate adaptation as a mechanism of adaptive resource management for wireless NCS. In digital control systems, the sampling rate specifies how often system components exchange information, and determines how well the digital controller approximates its continuous equivalent. Environmental disturbances can lead to system instability and cause uncertainty in system behavior. Intuitively,

This research was sponsored by the U.S. Army Research Office and Lockheed Martin and was accomplished under Cooperative Agreement W911NF10-1-0005. This work is also supported in part by the National Science Foundation under Grant CNS-1035655 and CCF-0820088. The views and conclusions contained in this document are those of the authors and should not be interpreted as representing the official policies, either expressed or implied, of the Army Research Laboratory or the U.S. Government. The U.S. Government is authorized to reproduce and distribute reprints for Government purposes notwithstanding any copyright notation hereon. the larger the sampling rate, the more frequent state updates the components of a NCS will receive, and thus can have a better ability to reduce the effect of such disturbances. On the other hand, sampling rates are naturally linked to the bandwidth demands of the network. From the network perspective, the sampling rates should be limited to avoid congestion and packet losses, which will deteriorate the NCS performance [1]. In a wireless network, where available bandwidth varies due to dynamic user behaviors and external interference sources, dynamic adaptation of sampling rates is needed to achieve desirable NCS performance and fully exploit the varying available bandwidth.

In this paper, we formulate the NCS sampling rate adaptation as an optimal resource allocation problem, where the NCS performance is maximized subject to the wireless bandwidth constraint. The key challenge is how to quantify the relationship between the NCS performance and its sampling rate. In this paper, we use a utility function to characterize this relationship, and formally define it as the ratio of a digital controller performance to the performance of its continuous equivalent. We use the noise covariance matrix of the control system to characterize system performance with respect to its ability of disturbance rejection. We show that this utility function is a strictly concave function of the sampling rate, when random white noise is considered as the model of control system disturbance. The concavity of the utility function reflects the marginal return on the NCS performance when its sampling rate further increases.

Based on this optimal rate allocation formulation, a pricebased algorithm is developed for distributed sampling rate adaptation. In this algorithm, a price is generated for each contention region of the wireless channel as a function of the sampling rate. The NCS then adapts its sampling rate based on its utility function so that its net profit, which is the difference between the utility and the cost (product of price and rate), is maximized. The stability of this algorithm is proved under our utility function model for networked control systems.

The main contributions of this paper are summarized as follows. First, we formalize the NCS performance based on its ability of disturbance rejection and derive a utility metric as a function of its sampling rate is a novel contribution. Traditionally, NCS operates at a fixed sampling rate based on its average or worst-case resource requirements. Recent work has identified the need for dynamic resource management of NCS [2][3][4], but the proposed approaches focus on off-line 
optimization. The approaches presented in [5][6][7] introduce methods for adapting sampling periods at run-time. However, their algorithms are based on fixed parameters and the effect of varying channel conditions are not considered. The works in [8] and [9] consider dynamic bandwidth allocation through sampling period adjustment for wireline networked control systems. In contrast, our work provides a fully distributed dynamic network resource management solution for wireless NCS. It fully exploits wireless network resources and maximizes the NCS performance. Second, the NCS sampling rate adaptation algorithm is evaluated in an integrated simulation environment [10] that consists of Matlab and ns-2. Using ns2 - a packet-level network simulator that implements all the details of the network protocol stack, allows highly accurate evaluation of network effects on the NCS performance, which is impossible by using Matlab/Simulink alone.

In Sec. II, we present the control system model and formally define the utility function to characterize the performance of a control system. In Sec. III, we present the wireless network model, formulate the problem of optimal rate allocation and derive the price-based rate adaptation algorithm. Finally we evaluate the algorithm using our Networked Control System Wind Tunnel (NCSWT) simulation environment in Sec. IV and conclude the paper in Sec. V.

\section{Control System Modeling}

In this section, we focus on the control system modeling and provide a formal description of the control system performance as a function of the sampling rate. First, we briefly describe the passivity based networked control architecture we used for our design. In sub-sections (A) and (B) we describe the continuous-time and discrete time control systems we used to determine our utility function which is described in sub-section (C). In Fig. 1, $G_{p}(s)$ is the plant system to be controlled.

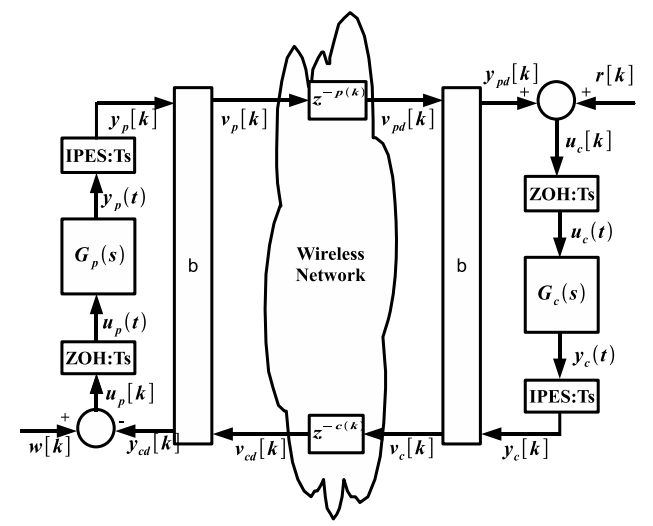

Fig. 1. Passivity Based Control Architecture Over Wireless Networks

The composite dynamics of the plant is by design passive, which means it ensures stability of the NCS in the presence of network uncertainties such as packet losses and time varying delays. The controller, $G_{c}(s)$ which is also passive, controls the plant to behave in a desired manner. The block $b$ transforms the power variables (i.e., the direct inputs and outputs of plant and controller) into wave variables for communication over a wireless network. These wave variables preserve the passivity of the transmitted information over the network. The inner product equivalent sampling (IPES) and zero order hold $(\mathrm{ZOH})$ blocks on both the plant and control sides are used to implement the passive discretization of the continuous time systems. We refer readers to [11] for a detailed description and proofs pertaining to the passive control architecture.

\section{A. Continuous-time Control System}

The continuous-time control system involves a continuoustime plant interacting with a continuous-time controller. The plant $G_{p}(s)$ is described by the following state-space representation.

$$
\begin{aligned}
& \dot{x}_{p}(t)=A_{p} x_{p}(t)+B_{p} u_{p}(t)+B_{w} w(t) \\
& y_{p}(t)=C_{p} x_{p}(t)
\end{aligned}
$$

where $x_{p}(t) \in \Re^{n}$ denotes the plant states, $u_{p}(t) \in \Re^{m}$ denotes the control input, $w(t) \in \Re^{m}$ is the disturbance input modeled as a zero-mean white noise process, and $y_{p}(t) \in \Re^{m}$ is the plant output. $A_{p}, B_{p}$, and $B_{w}$ define the plant state matrices and $C_{p}$ defines the plant output matrix.

The state space of the continuous-time controller $G_{c}(s)$ is

$$
\begin{aligned}
\dot{x}_{c}(t) & =A_{c} x_{c}(t)+B_{c} u_{c}(t) \\
y_{c}(t) & =C_{c} x_{c}(t)+D_{c} u_{c}(t)
\end{aligned}
$$

where $x_{c}(t) \in \Re^{n}$ denotes the controller state, and $u_{c} \in \Re^{m}$ denotes the error signal, or the difference between the plant output $y_{c}(t) \in \Re^{m}$ and the reference signal input $r(t) \in \Re^{m}$. $A_{c}$ and $B_{c}$ define the controller state matrices, while $C_{c}$ and $D_{c}$ define the controller output matrices.

From the plant and controller state-space description, the closed loop state space form of the system can be represented as follows:

$$
\begin{aligned}
& \dot{x}=\left[\begin{array}{c}
\dot{x}_{p}(t) \\
\dot{x}_{c}(t)
\end{array}\right] \\
& =\left[\begin{array}{cc}
A_{p}-B_{p} D_{c} C_{p} & B_{p} C_{c} \\
-B_{c} C_{p} & A_{c}
\end{array}\right]\left[\begin{array}{c}
x_{p}(t) \\
x_{c}(t)
\end{array}\right] \\
& +\left[\begin{array}{c}
B_{p} D_{c} \\
B_{c}
\end{array}\right] r(t)+\left[\begin{array}{c}
B_{w} \\
0
\end{array}\right] w(t) \\
& y=\left[\begin{array}{l}
y_{p}(t) \\
y_{c}(t)
\end{array}\right] \\
& =\left[\begin{array}{cc}
C_{p} & 0 \\
-D_{c} C_{p} & C_{c}
\end{array}\right]\left[\begin{array}{c}
x_{p}(t) \\
x_{c}(t)
\end{array}\right]+\left[\begin{array}{c}
0 \\
D_{c}
\end{array}\right] r(t)
\end{aligned}
$$

The covariance matrix of the zero-mean white noise process of the continuous system can be defined by

$$
E\left[w(t) w^{T}(t+\tau)\right]=Q \delta(\tau)
$$

where $E$ denotes the expected value and $Q$ represents the power spectral density of $w$, or the continuous-time noise covariance matrix. The power spectral density can also be referred to as the "white noise intensity" or mean-square spectral density. The continuous-time state covariance matrix $P_{c}$ can be described by

$$
P_{c}(t)=E\left[x(t) x^{T}(t)\right]
$$


Based on the knowledge of $Q$, the steady state value of the state covariance can be obtained by the equation [12]

$$
A_{c l} P_{c}+P_{c} A_{c l}+B_{w c l} Q B_{w c l}^{T}=0
$$

where the matrices $A_{c l}$ and $B_{w c l}$ denote the closed loop matrices, or the coefficients of $x(t)$ and $w(t)$ in Eq. (5) respectively. From the resulting state covariance matrix, the root mean square of a state can then be determined. The Root-Mean-Square (RMS) of the plant state is equivalent to the standard deviation. For example in the response to white noise, assuming the system has only one plant state variable $x_{p 1}$ and the result obtained for the plant state covariance is $v$. The RMS of the plant state will be equal to $\operatorname{sqrt}(v)$. If a plant has several states, we can use the one of the states as long as we compare it the same discrete state obtained from the minimal realization of the discretized continuous system.

\section{B. Discrete-time Control System}

The continuous-time control system is usually implemented as a discrete-time control system via discretization. The discretization is executed with a sampling time $T_{s}$ using an inner-product equivalent sample and hold (IPESH) transform in order to preserve the passivity properties of the system [13]. The resulting system is equivalent to a system with a discrete plant and discrete controller.

$G_{p}(z)$ represents the discrete-time equivalent of the continuous-time plant $G_{p}(s)$. The discrete-time state space of the plant can be given by

$$
\begin{aligned}
x_{p}(k+1) & =\Phi_{p} x_{p}(k)+\Gamma_{p} u_{p}(k)+\Gamma_{w} w(k) \\
y_{p}(k) & =C_{p d} x_{p}(k)+D_{p d} u_{p}(k)+D_{w} w(k)
\end{aligned}
$$

The state space of the discrete-time controller $G_{c}(z)$ equivalent to the continuous-time $G_{c}(s)$ can be given by

$$
\begin{aligned}
x_{c}(k+1) & =\Phi_{c} x_{c}(k)+\Gamma_{c} u_{c}(k) \\
y_{c}(k) & =C_{c d} x_{c}(k)+D_{c d} u_{c}(k)
\end{aligned}
$$

The overall closed-loop state equation can be determined from the discrete plant and discrete controller's state space representation. This can be described by

$$
\begin{aligned}
& x(k+1)=\left[\begin{array}{c}
x_{p}(k+1) \\
x_{c}(k+1)
\end{array}\right] \\
= & {\left[\begin{array}{cc}
\Phi_{p}-\Gamma_{p} D_{c d} S_{f} C_{p d} & \Gamma_{p} D_{c d} S_{f} D_{p d} C_{c d} \\
-\Gamma_{c} S_{f} C_{p d} & \Phi_{c}-\Gamma_{c} S_{f} D_{p d} C_{c d}
\end{array}\right]\left[\begin{array}{c}
x_{p}(k) \\
x_{c}(k)
\end{array}\right] } \\
+ & {\left[\begin{array}{c}
\Gamma_{p} D_{c d}\left(1-S_{f} D_{p d} D_{p c}\right) \\
\Gamma_{c}\left(1-S_{f} D_{p d} D_{c d}\right)
\end{array}\right] r(k) } \\
+ & {\left[\begin{array}{c}
\Gamma_{w}-\Gamma_{p} D_{c d} S_{f} D_{w} \\
-\Gamma_{c} S_{f} D_{w}
\end{array}\right] w(k) }
\end{aligned}
$$

where $S_{f}=\left(I+\left(D_{c d} * D_{c d}\right)\right)^{-1}$, and $I$ is the identity matrix.

Based on the knowledge of the continuous-time noise covariance matrix $Q$, the discrete-time noise covariance matrix $Q_{d}$ can be obtained using the Van Loan's algorithm [14] and can be defined as

$$
Q_{d}=\int_{0}^{T_{s}} \Phi(\tau) B_{w c l} Q B_{w c l}^{T} \Phi^{T}(\tau) d \tau
$$

The steady state discrete-time state covariance matrix can then be obtained from the following equation

$$
\Phi P_{d} \Phi^{T}+Q_{d}=P_{d}
$$

From the resulting state covariance matrix, the discrete RMS of the plant state can then be determined similar to the continuous-time case.

\section{Utility Function}

When the system is discretized using various sampling rates to implement a digital controller over a network or computer, the control system response to disturbances degrades compared to the continuous closed loop case. The level of degradation depends on the sampling rate. To characterize the impact of sampling rate on the disturbance rejection ability of a digital controller, we consider the RMS ratio of the discretetime system plant state and its continuous-time counterpart. Intuitively, increasing the sampling rate will decrease the RMS of the system's discrete plant state, where the continuous-time system establishes the lower bound of the RMS.

Formally, let's assume that the traffic from the controller to the plant shares the same data path as the traffic from the plant to the controller. Then a digital control system $f$ with sample size $p k t$ size and sample time $T_{s}$ will pose a bi-directional flow rate of $p_{f}=2 \times p k t \_s i z e / T_{s}$ to the network. We define the utility function of system $f$ as a function of rate $p_{f}$ as follows.

$$
U_{f}\left(p_{f}\right)=\frac{R M S_{\text {continuous }}}{R M S_{\text {discrete }}\left(1 / T_{s}\right)}
$$

Essentially, the utility function is established by comparing the performance of the discrete-time system with the continuous-time system. Using the continuous-time control system, we determine the ability of the control system to reject disturbance in the form of white noise. We then proceed to repeat a similar process when a digital implementation of the controller is used. This digital implementation depends on the chosen sampling rate. This utility function is essentially determined by the amount of degradation of the system response to white noise compared to the continuous closed loop system.

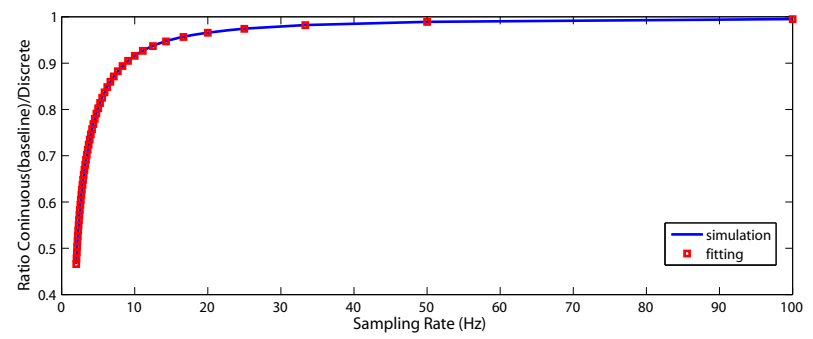

Fig. 2. System Utility Function

To demonstrate our utility function definition, we consider the following single-input-single output (SISO) linear-time invariant (LTI) system, without loss of generality and for simplicity. A plant system represents a single joint of a robotic arm, with the transfer function $G_{p}(s)=\frac{1}{J s}$. The transfer function of the controller is given by $G_{c}(s)=\frac{K_{p}+K_{d} s}{s}$. With 
the following parameters, $J=2.93, K_{d}=32.1$ and $K_{p}=8.2$. The utility function that characterizes the relationship between the sampling rate and this NCS system performance (as defined by the RMS ratio) is shown in Fig. 2. Fig. 2 shows that the utility function is strictly concave, with the red squares perfectly fitting the utility function. The fitted function is in the form of

$$
U_{f}\left(p_{f}\right)=\frac{p_{1} * p_{f}^{4}+p_{2} * p_{f}^{3}+p_{3} * p_{f}^{2}+p_{4} * p_{f}+p_{5}}{p_{f}^{4}+q_{1} * p_{f}^{3}+q_{2} * p_{f}^{2}+q_{3} * p_{f}+q_{4}}
$$

where $p_{1}, p_{2}, p_{3}, p_{4}, p_{5}, q_{1}, q_{2}, q_{3}, q_{4}$ are the fitted parameters.

\section{Optimal SAMPling RATE AdAPtation}

In this section, we first review the theoretical framework for optimal resource allocation in multi-hop wireless networks [15], and then present our price-based sample rate adaption algorithm.

\section{A. Wireless network model}

We consider a network that consists of a collection of wireless nodes $V$. Nodes within the transmission range of each other can communicate directly, forming a wireless link $l \in L$. While nodes that are far away communicate via relays of other nodes. Here we consider the protocol model [16] to characterize the location-dependent contention and spatial reuse of the wireless communication in this network. We further adopt the model presented in [15] where maximal cliques in the contention graph of a wireless network are used to characterize the independent resource elements in wireless networks. Here we denote a resource element as $e \in E$. Each resource element has a finite capacity $C_{e}$.

Such a network is shared by a set of control systems $F$. For each control system $f \in F$, its plant and controller are hosted on two different nodes in the network. We assume the traffic from the controller to the plant and the traffic backwards share the same network path. Then the control system $f$ generates a bi-directional flow between these two nodes. The flow may go through multiple hops in the network and traverse a sequence of resource elements. Let $R_{e f}$ be the amount of resource element $e$ used by system $f$.

\section{B. Optimal sampling rate allocation formulation}

The problem of optimal rate allocation in the sense of maximizing the aggregated utility of all control systems in the network can be formulated into the following nonlinear optimization problem:

$$
\begin{aligned}
& \mathbf{P}: \quad \text { maximize } \sum_{f \in F} U_{f}\left(p_{f}\right) \\
& \text { subject to } R \cdot p \leq C \\
& \boldsymbol{p} \geq 0
\end{aligned}
$$

In this formulation, the constraint (20) comes from the resource constraint of the shared wireless channel, where $\boldsymbol{R}=\left(R_{e f}\right)_{|E| \times|F|}$ is a matrix with element $R_{e f}$ at row $e$ and column $f$ [15]. $\boldsymbol{p}=\left(p_{f}, f \in F\right)$ and $\boldsymbol{C}=\left(C_{e}, e \in E\right)$ are vectors of flow rates and resource capacities respectively. By optimizing toward such an objective, the solution guarantees the optimal resource utilization.

\section{Price-based algorithm}

In the above formulation, the representation of the utility function is essential to further inspect the optimization problem and implement the price-based framework. We observe that the utility function of NCS defined in Sec. II is differentiable and strictly concave. Thus the objective function of $\mathbf{P}$ in Eq. (19) is differentiable and strictly concave. Further, the feasible region of the optimization problem in inequality (20) and (21) is convex and compact [15]. According to the non-linear optimization theory, unique optimal solution to the resource allocation problem $\mathbf{P}$ exists.

Now we consider the dual problem $\mathbf{D}$ of $\mathbf{P}$ using its Lagrangian form:

$$
\begin{aligned}
D(\boldsymbol{\mu})= & \mathbf{D}: \min _{\boldsymbol{\mu} \geq 0} D(\boldsymbol{\mu}) \\
= & \sum_{f \in F} \max \left(U_{f}\left(p_{f}\right)-p_{f} \sum_{e \in E} \mu_{e} R_{e f}\right) \\
& +\sum_{e \in E} \mu_{e} C_{e}
\end{aligned}
$$

$\boldsymbol{\mu}=\left(\mu_{e}, e \in E\right)$ is a vector of Lagrange multipliers, and may be interpreted as the implied cost, or penalty, of a subflow accessing the resource element $e$. In other words, $\mu_{e}$ is the shadow price of resource $e$. The price of a control system $f$, $\lambda_{f}=\sum_{e \in E} \mu_{e} R_{e f}$ can be interpreted as that system $f$ needs to pay for all the resources it uses. Note that for each wireless link, its price is the aggregated price of all the resources that it belongs to.

By solving the dual problem $\mathbf{D}$, the optimal rate for system $f$ can be derived from

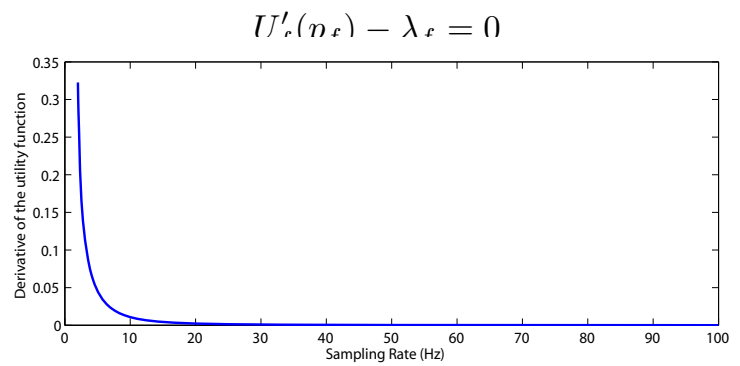

Fig. 3. Derivative of the Utility Function

Considering that the utility function is complicated as shown in Eq. (18), we use an approximated approach to solve $p_{f}$ from Eq. (24). Following the example in Sec. II, Fig. 3 represents the relationship between the sampling rate and price. By switching the $x$ and $y$ axes and curve fitting the plot, the sampling rate $p_{f}$ can be represented as a function of price $\lambda_{f}$

$$
p_{f}\left(\lambda_{f}\right)=a * \lambda_{f}^{b}
$$

where $a$ and $b$ are the fitted parameters. Thus the optimal rate for system $f$ can be approximated by substituting $\lambda_{f}$ into Eq.(25). 
Detailed derivation of $\boldsymbol{D}$ can be found in [15], while the adjustment of $\boldsymbol{\mu}$ can be represented as follows:

$$
\mu_{e}(i+1)=\left\lceil\mu_{e}(i)-\gamma\left(C_{e}-\sum_{e \in E} p_{f}\left(\lambda_{f}(i)\right) R_{e f}\right)\right\rceil^{+}
$$

Eq. (26) reflects the law of supply and demand. If the demand for channel $e$ exceeds its supply $C_{e}$, the resource constraint is violated. This will cause the increase of the channel price $\mu_{e}$. Otherwise, $\mu_{e}$ is reduced. The discrete time index $i$ in Eq. (26) is different from $k$ in the control system to ensure system convergence. As NCS is closed-loop, prices to controllers are appended onto the packets from the plants occupying only few bytes, and vise versa. This will not affect the overall network bandwidth much. Now we show the stability property of the price-based rate adaptation algorithm in the following theorem.

Theorem 1. Let $\mathcal{V}(\mu)$ be defined as

$$
\mathcal{V}(\mu)=\sum_{f \in F} \int_{0}^{\sum_{e \in f} \mu_{e}}\left(U_{f}^{\prime}(\eta)\right)^{-1} d \eta-\sum_{e \in E} \int_{0}^{\mu_{e}} q_{e}(\eta) d \eta
$$

$\mathcal{V}(\mu)$ is a strictly concave function and a Lyapunov function for the system of equations (24)-(26). The unique value $\mu$ maximizes $\mathcal{V}(\mu)$ and is also a stable point of the system where all trajectories converge.

Proof. We first will prove the strict concavity of the first term in $\mathcal{V}(\mu)$. As $U_{f}(\cdot)$ is strictly concave, its derivative $U_{f}^{\prime}(\cdot)$ exists and is strictly decreasing. Considering the utility function obtained in Sec. II, its derivative is shown in Fig. 3 as an example. Further we observe that $U_{f}^{\prime \prime}(\cdot) \leq 0$ is increasing. In addition, $\left(U_{f}^{\prime}(\cdot)\right)^{-1}$, the inverse function of $U_{f}^{\prime}(\cdot)$ has the same monotonicity as $U_{f}^{\prime}(\cdot)$. By integrating $\left(U_{f}^{\prime}(\cdot)\right)^{-1}$, the resulted function will have the same monotonicity as $U_{f}(\cdot)$, which is strictly concave. Further, as the definition domain of $U_{f}(\cdot)$ is the codomain of the inverse function of $U_{f}^{\prime}(\cdot)$, which is greater than 0 , the first term of $\mathcal{V}(\mu)$ is also greater than 0 .

Second, we prove the convexity of the second term in $\mathcal{V}(\mu)$. Let $q_{e}(\eta)=C_{e} \eta /(\eta+\varepsilon)$ [17]. It is a continuous and strictly increasing function of $\eta . q_{e}(\eta)$ arbitrarily closely approximates $C_{e}$ for a small positive $\varepsilon$. The strict concavity and positivity of the first term in $\mathcal{V}(\mu)$, as well as the assumptions on $q_{e}$ ensure that $\mathcal{V}(\mu)$ is strictly concave on $\mu \geq 0$ with an unique interior maximum $\mu$. It is determined by setting $\mathcal{V}^{\prime}(\mu)=0$.

$$
\begin{aligned}
\frac{\partial}{\partial \mu_{e}} \mathcal{V}(\mu) & =\sum_{e \in f}\left(U_{f}^{\prime}\left(\sum_{h \in f} \mu_{h}\right)\right)^{-1} R_{e f}-q_{e}\left(\mu_{e}\right) \\
& \geq \sum_{e \in f}\left(U_{f}^{\prime}\left(\sum_{h \in f} \mu_{h}\right)\right)^{-1} R_{e f}-C_{e}
\end{aligned}
$$

With Eq. (24), we have $\left(U_{f}^{\prime}\left(\lambda_{f}\right)\right)^{-1}=p_{f}$, so

$$
\begin{aligned}
\frac{d}{d t} \mathcal{V}(\mu(t)) & =\sum_{e \in E} \frac{\partial \mathcal{V}}{\partial \mu_{e}} \cdot \frac{d}{d t} \mu_{e}(t) \\
& \geq \gamma \sum_{e \in E}\left(\sum_{e \in f} p_{f}\left(\sum_{h \in f} \mu_{h}(t)\right) R_{e f}-C_{e}\right)^{2}
\end{aligned}
$$

This establishes that $\mathcal{V}$ is strictly increasing with $t$ unless $\mu(t)=\mu$, where the unique value $\mu$ maximizes $\mathcal{V}$. Thus function $\mathcal{V}$ is a Lyapunov function for the system (24)-(26), and the theorem follows.

\section{Performance Evaluation}

In this section, we evaluate the price-based sampling rate adaptation algorithm in the wireless networks using our Networked Control System Wind-Tunnel (NCSWT) environment [10]. NCSWT integrates two simulators Matlab and ns-2 based on HLA, which provides the standard for data communication and time synchronization of different simulation platforms. The tool allows us to simulate control system models in Matlab/Simlink and network models in ns-2. Using ns-2 - a packet-level network simulator that implements all the details of the network protocol stack, allows us to perform highly accurate evaluation of network effects on the NCS performance, which is impossible by using Matlab/Simulink alone.

\section{A. Simulation Setup}

In our experiment, the network system consists of three pairs of plants and controllers, all of which have the same utility function as presented in Section II. The wireless channel capacity of the wireless network is $1 M b p s$. The packet size is 500 bytes. Each simulation runs for 250 seconds.

The velocity of the plant system tracks a sinusoidal reference input $r[k]=\sin (\omega k)$ with $\omega=\frac{2 \pi}{40}$. The power spectral density of the white noise is set to 1 unless explicitly addressed. The sampling rate is adapted within the range of $[80,200]$ Kbps. $\gamma$ is set to 0.000000001 .

Three aspects of the system are examined:

1) performance of the control algorithm in terms of the convergence behavior of the plant output.

2) performance of the network in terms of its convergence behavior of the sampling rate.

3) performance of the overall NCSWT in terms of the difference between the plant output and the reference signal.

\section{B. Simulation Results}

1) Comparison with Fixed-Rate Control Systems: We first inspect how our price-based dynamical sampling rate adaptation algorithm performs. It is also compared with classical systems with fixed sampling rates. All the control systems share one channel. Fig. 4(a) shows the plant outputs of three
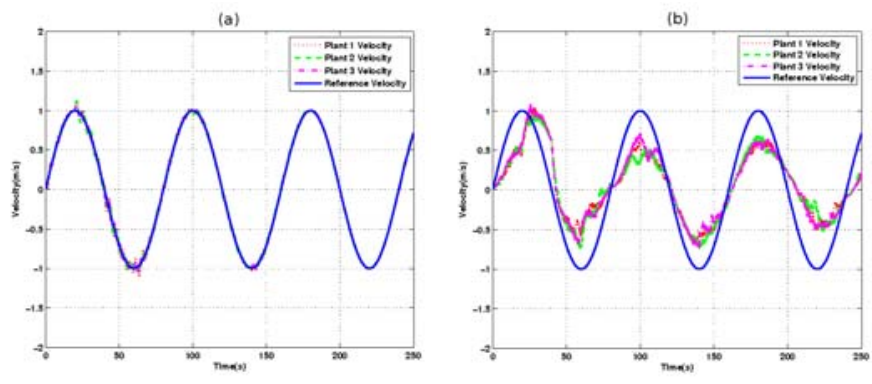

Fig. 4. Plant Outputs with Price-Based(a) and Sampling Time 0.01s(b) control systems. All the outputs closely follow the reference 
trajectory. Moreover, the differences between the plant outputs and the reference trajectory diminish with the increase of time. We then implement the classical rate allocation scheme with a fixed sampling rate different from the converged optimal sampling rate. Fig. 4(b) shows the plant outputs using fixed sampling times of $0.01 \mathrm{~s}$, corresponding to the rates of $100 \mathrm{Kbps}$. The outputs do not track the reference trajectory as well as those in Fig. 4(a). In Fig. 4(b), the sampling rate is larger compared with the converged optimal sampling rate of $80 \mathrm{Kbps}$, so the controller cannot be notified in time about the white noise disturbance to the system. This leads to larger tracking error and longer convergence time. In Fig. 4(b), the sampling rate is too large compared with the optimal value, and this rate causes congestion in the network. Many packets get lost during congestion, which leads to deteriorated system performance. In the passive system, it is exhibited as a decreased amplitude.

2) Impact of Dynamic NCS Join: NCS can use the converged optimal sampling rate as the fixed value when everything is static. However in practice, dynamics exists everywhere. For example, it is very common that NCS join or leave the network at run-time. A fixed sampling rate cannot be suitable for all the conditions. Thus it is important that an algorithm is able to dynamically reallocate network resources properly. In this experiment, with the three control systems
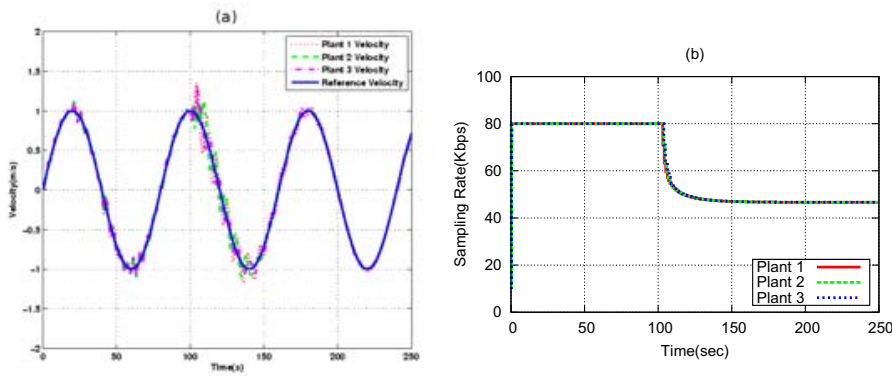

Fig. 5. Plant Output(a) and Sampling Rate(b) with Dynamic Join

start at the beginning, another two control systems join in at 100 second as background traffic. The two control systems use fixed sampling rate of $80 \mathrm{Kbps}$. Fig. 5 shows the plant outputs of the three control systems(a) and the variation of the sampling rates with time(b). At the beginning, the sampling rates quickly converge to $80 \mathrm{Kbps}$ with the plant outputs stabilize to the sinusoidal reference. When the background traffic joins in, the sampling rates re-converge to around $50 \mathrm{Kbps}$. The plant outputs experience certain period of oscillation before convergence. This is because that when the new systems join in, the network demand exceeds the channel capacity. In such a case, several packets get dropped due to queue loss, and the communication delay becomes pretty large until the law of demand and supply is satisfied again.

\section{CONCLUSION}

Sampling rate adaption is important for resource management of wireless networked control systems. In this paper we form a utility based optimization framework for rate allocation over wireless networks and present a distributed price based algorithm to solve the problem. With a utility function defining the relationship between the control system performance and the sampling rate, the algorithm fully exploits wireless network resources and maximizes the system performance. We perform simulation study using an integrated simulation tool where the control system is simulated in Matlab and the network model is simulated in ns-2. The experiment results show that our algorithm is able to provide agile and stable sampling rate adaptation for the networked control systems.

\section{REFERENCES}

[1] J. Bai, E. Eyisi, Y. Xue, and X. Koutsoukos, "Dynamic tuning retransmission limit of ieee 802.11 mac protocol for networked control systems," in Proc. of CPSCom, 2010, pp. 666-672.

[2] D. Setoand, J. P. Lehoczky, L. Shaand, and K. G. Shin, "On task schedulability in real-time control systems," IEEE Real-Time Systems Symposium, pp. 13-21, 1996.

[3] P. Martí, C. Lin, S. A. Brandt, M. Velasco, and J. M. Fuertes, "Draco: Efficient resource management for resource-constrained control tasks," IEEE Trans. on Computers, vol. 58, no. 1, 2009.

[4] L. Xiao, M. Johansson, H. Hindi, S. Boyd, and A. Goldsmith, "Joint optimization of communication rates and linear systems," IEEE Transactions on Automatic Control, vol. 48, no. 1, pp. 148-153, 2003.

[5] N. Ploplys, P. Kawka, and A. Alleyne, "Closed-loop control over wireless networks," IEEE Control Systems Magazine, vol. 24, no. 3, pp. 58-71, 2004.

[6] J. Colandairaj, G. Irwin, and W. Scanlon, "Wireless networked control systems with qos-based sampling," IET Control Theory and Applications, vol. 1, no. 1, pp. 430-438, 2007.

[7] M. Andersson, D. Henriksson, A. Cervin, and K. Arzen, "Simulation of wireless networked control systems," IEEE CDC and ECC, pp. 476-481, 2005.

[8] Z. Li and M. Chow, "Adaptive multiple sampling rate scheduling of real-time networked supervisory control system - part ii," IEEE IECON, pp. 4610-4615, 2006

[9] F. Xia, Y. Sun, and Y. Tian, "Feedback scheduling of priority-driven control networks," Comput. Stand. Interfaces, vol. 31, pp. 539-547, March 2009.

[10] D. Riley, E. Eyisi, J. Bai, X. Koutsoukos, Y. Xue, and J. Sztipanovits, "Networked Control System Wind Tunnel (NCSWT)- An evaluation tool for networked multi-agent systems," in Proc. of SIMUTools, 2011.

[11] N. Kottenstette, X. Koutsoukos, J. Hall, J. Sztipanovits, and P. Antsaklis, "Passivity-based design of wireless networked control systems for robustness to time-varying delays," in Proc. of RTSS, 2008, pp. 15-24.

[12] W. S. Levine, Control System Funcamentals. CRC Press, 2000.

[13] G. F. Franklin, J. D. Powel, and M. L. Workman, Digital Control of Dynamic Systems. Addison Wesley, 1997.

[14] N. Kottenstette, "Control of passive plants with memoryless nonlinearitites over wireless networks," Ph.D. dissertation, University of Notre Dame, 2007.

[15] Y. Xue, B. Li, and K. Nahrstedt, "Optimal resource allocation in wireless ad hoc networks: A price-based approach," IEEE Trans. on Mobile Computing, vol. 5, pp. 347-364, 2006.

[16] P. Gupta and P. Kumar, "The capacity of wireless networks," IEEE Trans. Information Theory, vol. 46, pp. 388-404, 2000.

[17] F. P. Kelly, A. K. Maulloo, and D. K. H. Tan, "Rate control for communication networks: Shadow prices, proportional fairness and stability," The Journal of the Operational Research Society, vol. 49, no. 3, pp. 237-252, 1998. 